\title{
Characterization of petrography and diagenetic processes influence on porosity and permeability of Oligocene sandstone reservoir rocks, block 15-2 in Cuu Long basin
}

\author{
Lieu, Kim Phuong ${ }^{1 *}$; Le, Thi Thu Hang ${ }^{1}$; Nguyen, Van Hieu ${ }^{1}$; Nguyen, \\ Minh Nhut ${ }^{1}$ \\ ${ }^{1}$ Analysis Laboratory Center-Vietnam Petroleum Institute, Vietnam
}

\begin{abstract}
Porosity and permeability are the two most important of reservoir rock characteristics particularly in sandstones. They are controlled by rock fabric, mineralogical composition, diagenetic evolution, etc. To address the unknowns, this investigation examined 98 of core, core plug and side wall core samples taken from oil fields of block 15-2 in Cuu Long basin. The samples were analyzed via thin section, Scanning electron microscope (SEM), X-ray diffraction (XRD) and measured helium porosity and Klinkenberg permeability. The results showed that most of sandstones in sequences BH1.2 and $\mathrm{C} 1$ are arkose and lithic arkose and sporadically interbedded by feldspathic greywacke and carbonate cemented sandstones while feldspathic greywacke and zeolite cemented sandstones are commonly present in sequences D1 and E1. The clean sandstones with moderately-well grain sorting have higher porosities and permeable abilities than the very poorly-poorly sorted sandstones. The medium-coarse grained sandstones have a higher porosity and permeability than the very finefine grained sandstones. The diagenetic processes of sandstones are progressively growing with the increasing burial depth as smectite transfers into illite and the amount of illite tends to increase while kaolinite reduces and transfers into dickite. Feldspars and lithic fragments are highly leached and sandstones are heavily restricted by cementation and compaction at deeper. The grain sorting, grain size, compaction and cementation cause to decrease the porosity and permeability of the sandstones. Additionally, clay minerals as illite and chlorite have a negative effect and cause highly decreasing permeability.
\end{abstract}

Keywords: Сии Long basin; sandstone reservoir; petrography; diagenetic processes; porosity and permeability

\section{INTRODUCTION}

Petrography characteristics as mineralogical composition, grain texture and lithic formation are significant factors owing to their influences on the porosity and permeability of sandstone reservoir rocks. These are important parameters that control how much hydrocarbon a formation can store and pass through.

Previous investigations in terms of grain size, grain sorting, spatial distribution and diagenetic features that control porosity and permeability of sandstones have been done by some researchers such as Worden (1998) and Morad (1998). Effect of sorting on porosity was investigated by Bear (1972). Variation of porosity and permeability due to influence of grain size, sorting, cement volume was showed by McKinley, et al (2011). The relationship between grain size and reservoir rock characteristics was demonstrated by Griffith (1952).

In this work, the article reveals petrography features, post-depositional diagenesis and gives factors that impact on porosity and permeability of
Oligocene sandstones, block 15-2 in Cuu Long basin.

Cuu Long basin is a Tertiary rift basin, located on the southern shelf of Vietnam. The northern west of Cuu Long basin is bordered by continental block. To the southern west the basin is bordered by Khorat-Natuna swell and to the northern east it is bordered by Tuy Hoa fault. The south and southeast of Cuu Long basin is Con Son swell. It covers on an area of roughly $36,000 \mathrm{~km}^{2}$ consisting blocks: 01, 02, 09, 15-1, 15-2, 16 and 17 in which block 15-2 is on the west of the basin (fig. 1).

The basin has been filled by Tertiary sediments and has the largest oil reserve in Vietnam. Oil has been exploited from Early Miocene and Oligocene sandstone reservoirs of blocks: 15-2; 151 ; 01; 09-2; 09-1 16-1. Oligocene stratigraphy divided into Upper Oligocene characterized by Tra Tan formation, which are composed of sequences $\mathrm{C} 1$ and D1 and Lower Oligocene characterized by Tra $\mathrm{Cu}$ formation, which is included of sequences E1 and F1 (Tran Le Dong and Phung Dac Hai, 2005). 


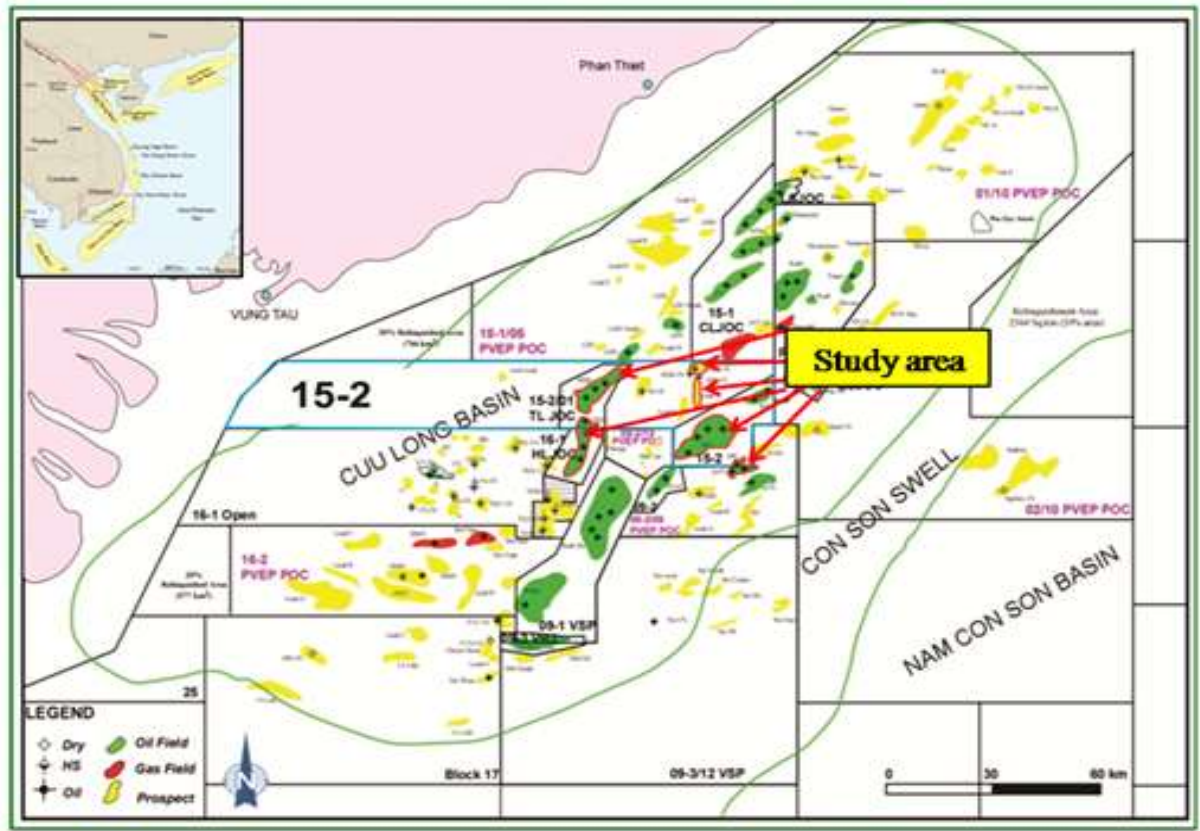

Figure 1. Map of study area (Petro Vietnam 2014)

Recently studies detected out palynomorph assemblages as Verrutricolporites pachydermus, Jussiena spp., Cicatricosisporitesdorogensis in sequence BH1.2 that is featured in Lower MioceneUpper Oligocene (?) (Mai Hoang Dam and Chu Duc Quang, 2015). Oligocene sandstone sequences in block 15-2 are distinct petrographic characterization and have experienced diagenetic processes. Hence the porosity and permeability of each sandstone sequence are heterogeneous and resulting to its reservoir quality is not being the same.

\section{METHODS}

To carry out the investigation, 98 of core, core plug and side wall core samples were taken from oil fields in Oligocene sandstone reservoirs of block 15-2 in Cuu Long basin. All samples were analyzed by thin section under microscope as grain size measure (Wentworth's scale, 1922); in terms of grain texture description including grain roundness, grain sorting, arrangement and grain contacts (Folk, et al., 1970) and (Petijohn et al., 1987). Verifying rock composition and porosity is carried out by mineral point counting method and rock classification is based on the mineralogical composition of their framework grains, which are quartz, feldspar, lithic fragments (Folk's classification, 1974). Clay fraction with the particle size smaller than $2 \mu \mathrm{m}$ was determined by XRD analysis via D8-ADVANCE and calculated amount of semi-quantitative by Topas software. Clay mineral morphologies, their distribution, arrangement in pore spaces and relation to framework grains were verified by SEM examination (Welton, 2003). Then the samples were measured helium porosity as Boyle's law and Klinkenberg permeability as Darcy's law. Basing the analytic results, the assessment of reservoir rocks is according to Levorsen (1967).

\section{RESULTS}

\section{III.1. Petrographic characterization}

Oligocene sandstone reservoir rocks in block 15-2 consist of sequences BH1.2, C1, D1, E1 and $\mathrm{F} 1$ that are made up of many types of sandstones having the grain size variation from very fine to coarse and very coarse, locally gravel and pebble. The textural characteristics of sandstone as grain sorting is poor to good, less frequently very good, grain roundness is commonly angular, sub-angular, sub-rounded, less commonly rounded and grain contacts are point to point, long and concavoconvex, rarely floating. Almost all Oligocene reservoir sandstones are clean and commonly rich of quartz, feldspar and granitic fragments; locally, including metamorphic and volcanic debris (fig. 2a). Sporadically carbonate cemented sandstone and matrix-rich sandstone, larger than $15 \%$ interbed in clean sandstones (fig. 2b\&c). Being noted that matrix-rich sandstones frequently occur in sequence D1 and the sandstones that are principally cemented by zeolite are richly present in sandstones of sequence E1 (fig. 2d). The major authigenic and cement minerals are clays with subordinate quartz overgrowths, albite, siderite and opaque minerals and distributed dominantly in sequences BH1.2 and $\mathrm{C} 1$. The porosity and permeability of sandstones are in a wide ranging from very poor up to good and very good. Their porosity and permeability are varying due to influence of grain sorting, grain size 
between oil fields after deposited sediments and owing to impact of cementation, mechanic compaction along with the burial depth. There is none core samples taken from sequence F1 so this work does not consider. Petrographical features, diagenetic processes and correlation of porosity and permeability of each sequence are mentioned in more detail below.

\section{Sequence BH1.2:}

The sandstones are composed of very fine to coarse and very coarse grains and are determined mainly as arkose, lithic arkose with lesser matrixrich feldspathic greywacke. The grain roundness is angular, sub-angular to sub-rounded and the grain contacts are dominantly point to point and long types. Medium to coarse and very coarse grained sandstones are very poorly, poorly and moderately sorting. Very fine to fine grained sandstones have the better grain sorting. The grain sorting is dominantly moderate, moderately good-good and rarely poor. The framework grains are cemented predominant clays and subordinate carbonate as calcite and quartz overgrowths, which are sporadically surrounding on quartz grain surfaces. The porosity and permeability of moderately sorted, coarse and very coarse grained sandstones are the largest and of poorly sorted, fine grained sandstones are the smallest (tab. 1). It reveals the porosity and permeability of sandstones are significantly depending on grain sorting and grain size. Less common feldspathic greywacke sandstones with rich of matrix have very poor porosity that is almost smaller than $5 \%$.

\section{Sequence C1:}

Most of sandstones are clean and matrixpoor, classified as arkose and lithic arkose. Rarely occurrence of poikilotopic carbonate cemented sandstone is in the sequence. The sandstones contain sub-angular to sub-rounded framework grains, less frequently angular and rounded grains. Coarse-very coarse grained sandstones are very poorly-poorly sorted and fine-medium grained sandstones are poorly-moderately sorted. The contacts of framework grains are point to point and long types. The authigenic and cement minerals are predominantly clays, less frequently carbonate, albite and quartz. The porosity of fine-medium grained sandstones is higher compared to that of coarse-very coarse grained sandstones and poikilotopic carbonate cemented sandstone whereas fine grained sandstones have the lowest permeability (tab. 2). Their porosities seriously depend on the grain sorting.

\section{Sequence D1:}

Sandstones are built up by very fine to fine grains and some are medium-coarse grains. The roundness of grains is sub-angular, sub-rounded, locally rounded. The matrix-poor sandstones are determined as arkose and lithic arkose with moderately-well sorted and the matrix-rich sandstones are classified as feldspathic greywacke with poorly sorted that are interbedding in arkose and lithic arkose. The framework grains of sandstones are mainly cemented by clays and carbonate minerals as calcite, siderite and dolomite and authigenic quartz that grows on the detrital quartz surface. Carbonate minerals and quartz overgrowths just appear in local. The porosity of feldspathic greywacke sandstones is none appearance due to matrix pore-filling. The clean medium-coarse grained sandstones have the moderate-good porosity and the good permeability while the poor-very poor porosity and permeability are noted in the very fine-fine grained sandstones (tab. 3). It demonstrates that the porosity and permeability downgrade with decreasing the grain size.

\section{Sequence E1:}

The sandstones are composed of mediumcoarse grained sandstones, which are interbedded by very fine-fine grained sandstones. Locally, there is the appearance of very coarse sandstones and gravels, pebbles with granular shape, which are very poorly sorted. The sandstones are strongly squeezed as showing the grain contacts is long and concavoconvex types. The grain sorting of the sandstones is commonly moderate to good and the framework grain roundness is sub-angular, sub-rounded to rounded, sometimes the medium-coarse sandstones contain the angular grains. Most of sandstones are clean, poor matrix and classified arkose and lithic arkose, rarely the matrix-rich sandstones are feldspathic greywacke. The sandstones are cemented by clays with subordinate by carbonate minerals as calcite and siderite in local calcite presents as poikilotopic cement. Particularly, the sandstone in this sequence was directly deposited on igneous basement rock and influenced by magma activity. Hence the sandstone sequence was crossed by extrusive rock dykes and the sandstones were cemented by zeolite that occurs quite abundantly. The porosity of the arkose and lithic arkose sandstones is related to the grain size and grain sorting as showing the moderately-well sorted, coarse sandstones has a higher visible porosity compared to the poorly sorted coarse sandstones and medium sandstones. The fine sandstones are in very poor visible porosity (tab. 4). The porosity of the zeolitized sandstones significantly depends on the amount of zeolite appearing in the sandstones (tab. 4). The highly zeolite cemented sandstones have low pore spaces between framework grains. 
Table 1. Summary of petrographic data and porosity, permeability of sequence BH1.2

\begin{tabular}{|c|c|c|c|c|c|c|c|c|c|c|c|c|c|c|c|c|c|}
\hline \multirow{2}{*}{$\begin{array}{l}\text { Tpe } \\
\text { of } \\
\text { mis }\end{array}$} & \multicolumn{5}{|c|}{ Tentre: } & \multirow[t]{2}{*}{ fage: } & \multicolumn{3}{|c|}{ Brasodidens (2) } & \multirow{2}{*}{$\frac{\operatorname{lagam}(\%)}{C_{7 f}}$} & \multicolumn{4}{|c|}{ 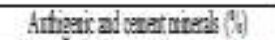 } & \multirow{2}{*}{$\begin{array}{l}\text { Vablepurt } \\
\text { oums } \\
\text { Ponos fis }\end{array}$} & \multirow{2}{*}{ 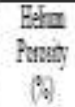 } & \multirow{2}{*}{$\begin{array}{c}\text { Noridy? } \\
\text { Pansithy } \\
\text { (ID) }\end{array}$} \\
\hline & $\begin{array}{l}\text { Grim } \\
\text { ife }\end{array}$ & Sictur & $\begin{array}{l}\text { Stadiat } \\
\text { drimon }\end{array}$ & $\begin{array}{l}\text { Gran } \\
\text { mats: }\end{array}$ & $\begin{array}{l}\text { Gran } \\
\text { contos }\end{array}$ & & 5 & Q & ? & & $d \pi$ & $\begin{array}{c}\text { Cabrat } \\
\text { e }\end{array}$ & Qhen & $\begin{array}{l}\text { Opars: } \\
\text { roxal }\end{array}$ & & & \\
\hline \multirow{14}{*}{$\begin{array}{l}\text { fins } \\
\text { tad } \\
\text { Ifris } \\
\text { atsis }\end{array}$} & \multirow{6}{*}{$\begin{array}{l}\text { Carse } \\
\text { adrey } \\
\text { coser }\end{array}$} & \multirow[t]{2}{*}{$\mathbb{P}$} & \multirow[t]{2}{*}{202.52} & \multirow[t]{6}{*}{ ASASB. } & \multirow[t]{6}{*}{ P. } & IIn:lla & 55 S.S.1.4 & $+49-48.8$ & [3-3.7. & Q12.4 & 2117 & 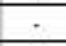 & - & - & $566^{\circ}$ & - & . \\
\hline & & & & & & Connsy & + & $\cdot$ & - & 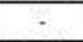 & 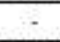 & . & 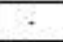 & - & - & - & 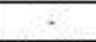 \\
\hline & & \multirow[t]{2}{*}{ P } & \multirow[t]{2}{*}{ 1X:199 } & & & 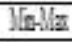 & 35.521 & 270.615 & 5.57 & 1078 & 2431 & M.1.10 & 0.28 & 0066 & 3250 & 2123.7 & 107.19421] \\
\hline & & & & & & Comen & 46647 & $494+6$ & 81.119 & . & 23.132 & . & 112.28 & 020.3 & $18+401$ & - & B68.-1992. \\
\hline & & \multirow[t]{2}{*}{ II } & \multirow{2}{*}{0771.18} & & & Jolles & 495010 & 365.31 .1 & $119-13.6$ & - & 3640 & MA. & 32.14 & 020.4 & 182304 & 10.9.4 & SQLPTHO \\
\hline & & & & & & $\operatorname{Conn}$ & 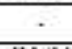 & 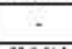 & - & 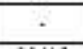 & 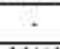 & . & 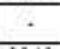 & - & - & 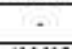 &. \\
\hline & \multirow[t]{4}{*}{ Jefirs } & \multirow[t]{2}{*}{$P H$} & \multirow[t]{2}{*}{191.1 .88} & \multirow[t]{4}{*}{ ASASS } & \multirow[t]{4}{*}{ PL } & $\mathrm{J}[\mathrm{a}) / \mathrm{ax}$ & $322-426$ & 3266010 & 0.1 .173 & 0.115 & 26:56 & $\mathrm{Maj} 3$ & 0.40 & 00.11 & 38.166 & 199807 & 502100 \\
\hline & & & & & & Conny & 4.6469 & 376594 & 169416 & 79.90 & 40115 & - & 16.38 & 03.47 & 12417.4 & - & 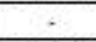 \\
\hline & & \multirow[t]{2}{*}{ WLG } & \multirow{2}{*}{0522.7} & & & Jallas & 32.45 & 310.523 & {$[+22]$} & 24.6 & 40.14 & 1046 & 3.40 & 02.06 & $160 .-18.6$ & 20.12 & 4.7250 \\
\hline & & & & & & Cotans & 4.2450 & +1..5.23 & 11391 & 16.7 & 62.122 & 12.1 .4 & 34.38 & 02.24 & 16217.6 & 31.200 & $450260)$ \\
\hline & \multirow[t]{4}{*}{ Fae } & \multirow[t]{2}{*}{ ? } & \multirow[t]{2}{*}{10.15} & \multirow[t]{4}{*}{ ASASBR. } & \multirow[t]{4}{*}{ PLL } & J[allar & 38541. & 51.550 & 579.7 & $2+131.4$ & 36.5 & . & $2+\sqrt{11}$ & - & $20-3.4$ & - & + \\
\hline & & & & & & Coming & & - & . & & - & - & $\cdot$ & - & 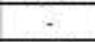 &. & - \\
\hline & & \multirow[t]{2}{*}{ WHG } & 184.7\% & & & 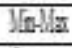 & 32.123 & 310.519 & 8.2 .11 & 1213.4 & 367 & SDIO & $2+.14$ & 0.001 & $20 \% 2$ & & - \\
\hline & & & & & & Coming & $324 t 3$ & 492516 & 97.197 & 56121 & $5+6.4$ & 1210 & $1+28$ & - & 112172 & & - \\
\hline
\end{tabular}

Note: VP: very poor; P: poor; M: moderate; MG: moderate good; A: angular; SA: sub-angular; SR: subrounded; $P$-L: point-long

$Q$ : all quartz but not quartzite; $F$ : feldspars and granite fragments; $R$ : Other rock fragments; in this study, $R$ consists mainly of volcanic and metamorphic fragments); Visible porosity is not including micropores.

Table 2. Summary of petrographic data and porosity, permeability of sequence $\mathrm{C} 1$

\begin{tabular}{|c|c|c|c|c|c|c|c|c|c|c|c|c|c|c|c|c|c|}
\hline \multirow{2}{*}{$\begin{array}{l}\text { Type } \\
\text { of nock }\end{array}$} & \multicolumn{5}{|c|}{ Tentre } & \multirow{2}{*}{ Bang: } & \multicolumn{3}{|c|}{ Franemod grans $(2 a)$} & \multirow{2}{*}{ 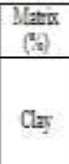 } & \multicolumn{4}{|c|}{ Auttigaic ad senert tinerls (h) } & \multirow{2}{*}{ 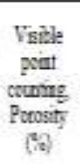 } & \multirow{2}{*}{$\begin{array}{l}\text { Helinm } \\
\text { Ponsity } \\
(69)\end{array}$} & \multirow{2}{*}{$\begin{array}{l}\text { Krolaberg } \\
\text { Pemeakilty } \\
\text { (ID) }\end{array}$} \\
\hline & $\begin{array}{l}\text { Gram } \\
\text { sige }\end{array}$ & Sorting & $\begin{array}{l}\text { Stadard } \\
\text { deriation }\end{array}$ & $\begin{array}{c}\text { Grain } \\
\text { swothess }\end{array}$ & $\begin{array}{l}\text { Grin } \\
\cos =0\end{array}$ & & $\mathrm{~F}$ & $Q$ & $\mathrm{R}$ & & Clay & Caboute & Quate & $\begin{array}{l}\text { Oparez } \\
\text { minerds }\end{array}$ & & & \\
\hline \multirow{10}{*}{$\begin{array}{l}\text { Aitose } \\
\text { sid } \\
\text { intac } \\
\text { ainse }\end{array}$} & \multirow{4}{*}{$\begin{array}{c}\text { Coarse } \\
\text { and } \\
\text { very } \\
\text { ovase }\end{array}$} & \multirow{2}{*}{ IP } & \multirow{2}{*}{202.23} & \multirow{4}{*}{ ASA-SRR R } & \multirow{4}{*}{ P.I } & Drastas & $345+1.9$ & 572.622 & $1.3-33$ & 5.4 .65 & 21.137 & 1417.5 & & 00.26 & 2228 & - & - \\
\hline & & & & & & Camser & - & - & - & . & - & - & - & - & - & - & - \\
\hline & & \multirow{2}{*}{ P } & \multirow{2}{*}{$1 ; 34.1 .42$} & & & Strulas & 48.629 & 231.35 .3 & $11.6 \times 2.4$ & 3.0.5.0 & 32.50 & 0.40 & 20.68 & 00.0 .6 & 92-13.4 & 148.179 & W5R-8740 \\
\hline & & & & & & Camser & . & - & - & . & - & - & - & . & - & - & - \\
\hline & \multirow{4}{*}{ Meding } & \multirow{2}{*}{$\mathrm{P}$} & \multirow{2}{*}{ 140-1.16 } & \multirow{4}{*}{ ASA-SRR R. } & \multirow{4}{*}{ P.I } & Mens:las & 440.525 & 31.555 .1 & $16,2-201$ & 0007.8 & 42.9 .5 & 0.0 .1 .4 & 004.6 & 00.6 & 24162 & 18.0.19.4 & 2100.85220 \\
\hline & & & & & & Comosn & 445.525 & $315-365$ & 16:-19.0 & 0.4 .52 & 4246 & - & $30-46$ & 0.4 .6 & $14+162$ & 18.0-19.1 & $420.0-7620$ \\
\hline & & \multirow{2}{*}{ M } & \multirow{2}{*}{0.71 .091} & & & 15axyax & 415.503 & 342-3999 & $123-241$ & . & 40.64 & 0.0 .0 .8 & 46.60 & 0.4 .66 & 152.210 & 18.8-23.1 & 32000-9610 \\
\hline & & & & & & Cemssa & 478.503 & 345.399 & 139.241 & - & 40.6 .4 & 02.08 & $46-5.4$ & - & 172.201 & $203,23,1$ & 5970.8992 \\
\hline & \multirow{2}{*}{ Fas } & \multirow{2}{*}{ P-SE } & \multirow{2}{*}{ 08:1.13 } & \multirow{2}{*}{ ASAS-SR.R. } & \multirow{2}{*}{ P.I } & Bin-Mars & $33.5+6.5$ & 371400 & $16+278$ & 10.20 & 28.50 & 0.424 & $42-68$ & 0.620 & 14.4 .30 .4 & 195.21 .5 & 331403.0 \\
\hline & & & & & & Comson & 38.646 .5 & 387480 & 21.4 .278 & - & 36.50 & - & 46.68 & 10.20 & 170.004 & 20.021 .5 & $1240-403.0$ \\
\hline
\end{tabular}

Table 3. Summary of petrographic data and porosity, permeability of sequence D1

\begin{tabular}{|c|c|c|c|c|c|c|c|c|c|c|c|c|c|c|c|c|c|}
\hline \multirow[b]{2}{*}{$\begin{array}{l}\text { Inpec } \\
\text { ofrick }\end{array}$} & \multicolumn{5}{|c|}{ Timtar } & \multirow[b]{2}{*}{ Ravy } & \multicolumn{3}{|c|}{ 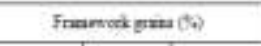 } & \multirow{2}{*}{ 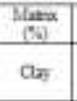 } & \multicolumn{4}{|c|}{ 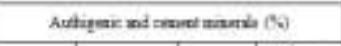 } & \multirow{2}{*}{ 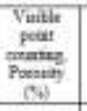 } & \multirow{2}{*}{ 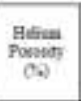 } & \multirow{2}{*}{ 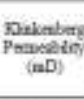 } \\
\hline & Gaie & sonuz & $\begin{array}{l}\text { Strodad } \\
\text { dthintiat }\end{array}$ & $\begin{array}{l}\text { Enan } \\
\text { nanatums }\end{array}$ & $\begin{array}{l}\text { Gon } \\
\operatorname{coth} t\end{array}$ & & $F$ & Q & P. & & $\mathrm{Cl}_{x y}$ & Cabcuar: & Quret & $\begin{array}{l}\text { Opage } \\
\text { wanth }\end{array}$ & & & \\
\hline \multirow{4}{*}{ 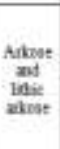 } & \multirow{2}{*}{ Mustax } & \multirow{2}{*}{$M 300$} & \multirow{2}{*}{ Q5.1 } & \multirow{2}{*}{ SA SBR } & \multirow{2}{*}{ PI } & Manster & 414.225 & 3atuss & 199.188 & 20.04 & 12.94 & 00.16 & 12.30 & 00.00 & 6.464 & 122,124 & 0928899. \\
\hline & & & & & & Coumese & 46.5250 & 329.52 .4 & $56 \div 18.8$ & - & 80.91 & - & 20.32 & & 130.6164 & 143-16.1 & 129.895 \\
\hline & \multirow{2}{*}{ foster } & \multirow{2}{*}{$M \cap \infty$} & \multirow{2}{*}{$0 \leq 0,08$} & \multirow{2}{*}{ SASRER } & \multirow{2}{*}{ PLC } & Meeman & 135.370 & 40.0 .61 .5 & 19953.334 & 76.11 .6 & $26-130$ & $12: 42$ & 12.58 & $0.0-1.2$ & 0.032 & $3+52$ & $\infty 0001$ \\
\hline & & & & & & Courase & 247.520 & 43542.6 & 293.35 .4 & 52.1116 & 20.94 & $=32.122$ & 20.58 & 0.8 .12 & - & 48.52 & - \\
\hline
\end{tabular}

Note: VP: very poor; P: poor; M: moderate; MG: moderate good; A: angular; SA: sub-angular; SR: subrounded; $R$ : rounded; $P-L-C$ : point-long and concavo-convex; $Q$ : all quartz but not quartzite; F: feldspars and granite fragments; $R$ : Other rock fragments; in this study, $R$ consists mainly of volcanic and metamorphic fragments); Visible porosity is not including micropores. 
Table 4. Summary of petrographic data and porosity, permeability of the clean sandstones in sequence E1

\begin{tabular}{|c|c|c|c|c|c|c|c|c|c|c|c|c|c|c|c|c|c|c|c|}
\hline \multirow{2}{*}{$\begin{array}{c}\text { Type of } \\
\text { nock }\end{array}$} & \multicolumn{5}{|c|}{ Textare } & \multirow{2}{*}{ Rarge } & \multicolumn{3}{|c|}{ 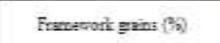 } & \multirow{2}{*}{\begin{tabular}{|c|}
$\begin{array}{c}\text { Marix } \\
(\% / 9)\end{array}$ \\
Clay
\end{tabular}} & \multicolumn{6}{|c|}{ Authigencic and censat mirenals (5) } & \multirow{2}{*}{$\begin{array}{l}\text { Tisible point } \\
\text { corminge } \\
\text { Perosity (20) }\end{array}$} & \multirow{2}{*}{$\begin{array}{l}\text { Heling } \\
\text { Posersity } \\
\text { (35ig) }\end{array}$} & \multirow{2}{*}{$\begin{array}{l}\text { Klinkenberg } \\
\text { Pemeability } \\
\text { \{nD) }\end{array}$} \\
\hline & $\begin{array}{l}\text { Grain } \\
\text { sira }\end{array}$ & Soeting & $\begin{array}{l}\text { Sardard } \\
\text { devisfoa }\end{array}$ & $\begin{array}{c}\text { Gain } \\
\text { nocuthess }\end{array}$ & $\begin{array}{l}\text { Greir } \\
\text { coetser }\end{array}$ & & F & Q & R & & Clay & Carboase & Quartr & $\begin{array}{l}\text { Opagua } \\
\text { minerals }\end{array}$ & Albite & Zeolite & & & \\
\hline \multirow{10}{*}{$\begin{array}{c}\text { Arkase ard } \\
\text { latis: } \\
\text { extose }\end{array}$} & \multirow{4}{*}{ Cousta } & \multirow{2}{*}{ P } & \multirow{2}{*}{$125 \cdot 1.4$} & \multirow{2}{*}{ A.SA-SR } & \multirow{2}{*}{ L.C } & Max-Mex & 382429 & $36.4-365$ & $20.6-25.4$ & - & 6.0 .72 & $3.2-58$ & $64-6.6$ & $0.6-9.8$ & $0.6-1.5$ & - & 126.3 & 6.6 .8 .3 & $0.00056-00006$ \\
\hline & & & & & & Corment & - & - & - & - & - & - & - & - & - & - & - & - & - \\
\hline & & \multirow{2}{*}{ WMSG } & \multirow{2}{*}{$0.58-1.9$} & \multirow{2}{*}{ A-SA-SR } & \multirow{2}{*}{ L-C } & Nr-Max & $412-633$ & $36.7-538$ & $0.4-13$ & $20-14$ & $5.0-10.2$ & 0.046 & $0.0-1.3$ & $00-12$ & - & - & $3.4-11.6$ & - & - \\
\hline & & & & & & Cormans & 49.1 .59 .6 & $37.0-501$ & - & - & $3.5-7.6$ & $266-6.2$ & - & - & - & - & 3.4 .5 .4 & - & - \\
\hline & \multirow{2}{*}{ Madine } & \multirow{2}{*}{ MLSIGG } & \multirow{2}{*}{$0.52-4.76$} & \multirow{2}{*}{$A S A-3 R \cdot R$} & \multirow{2}{*}{ PL-C } & 15r-3ax & $35.0-457$ & 39.741 & 172.162 & - & 5.3 .9 .8 & 3.4 .88 & 44.93 & 0.450 & $00-12$ & - & 0.45 .3 & 5.6-10.6 & $0.0025-0.05312$ \\
\hline & & & & & & Crmmen & - & - & - & - & - & - & - & - & - & - & - & - & - \\
\hline & \multirow{4}{*}{ Fine } & \multirow{2}{*}{ MSUSG } & \multirow{2}{*}{0.64 .8 .8} & \multirow{2}{*}{$5 .-S R \cdot R$} & \multirow{2}{*}{ PL-C } & 15r-Mas & $273-773$ & $20.5-70.1$ & $0.0-10.2$ & $0.4-16$ & 0.6 .5 .8 & $0.0-6.4$ & $0.0-2.7$ & $00-0.8$ & - & - & $0.0-52$ & - & - \\
\hline & & & & & & Common & - & - & - & - & - & - & . & - & - & - & - & - & - \\
\hline & & \multirow{2}{*}{ P.SI } & $08 \mathrm{OS}$ & $=$ & DT & 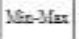 & 321.439 & $32.8-418$ & $18.4-351$ & $0.8-16$ & $6:-11.4$ & 4.296 & 4.4 .73 & $0.6-1.6$ & $0.4-12$ & - & 0.04 .8 & $66-102$ & $2.0016-000008$ \\
\hline & & & & & & Consaro & - & - & - & - & - & - & . & - & - & - & - & . & - \\
\hline & & & & & & Mar-min & $43.7-63.3$ & $36.7-563$ & $8.0-6.4$ & $0.0-5.0$ & $2.6-8.2$ & 1.0 .8 .4 & $0.0-12$ & $00-12$ & - & $14.6-291$ & $15-5.4$ & $4.8-13.9$ & 0.01-16.7 \\
\hline & & & & & & Comares & $491-59.4$ & $44.5-541$ & 27.50 & $20-12$ & $3.6-6.1$ & $1.2-32$ & - & - & - & $13.0-248$ & $22-6.0$ & 52.9 .4 & $0.4-14.2$ \\
\hline Sedstosa & & & & & & 3 las $-\min$ & $480-680$ & $36.8-520$ & $0.0-9$ & 0.40 & 0.5 .82 & 1.2 .5 .4 & $0,0.8$ & 0.2 .22 & - & $13.1-350$ & 20.7 .6 & $12-14.5$ & $0.001-10.00$ \\
\hline by zaolits & & & & & & Conmen & $525-405$ & $43.2-521$ & $0 .-31$ & $0.8-12$ & $2.4-6.0$ & $1.8-3.4$ & - & $0.4-1.0$ & - & 21:1-30.4 & $28-5.0$ & $3.8-8.6$ & $0.04-7.6$ \\
\hline & $=i n$ & Me & $0: 077$ & 840078 & $1 \mathrm{C}$ & Marsin & $170-770$ & $21.0-360$ & $00-100$ & a.9-20 & $0.5-6.0$ & $0.0-6.4$ & $0.0-2.7$ & 000.3 & - & $11-42$ & $17-5.2$ & 09.59 & $0.01-0.73$ \\
\hline & - & 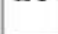 & (2) & (2) & & Canmano & - & - & - & - & + & - & . & - & - & & - & - & $=$ \\
\hline
\end{tabular}

Note: P: poor; M: moderate; MG: moderate good; G: good; A: angular; SA: sub-angular; SR: sub-rounded; R: rounded; $L-C$ : long-concavo-convex; P/L-C: long-concavo-convex and sometime point; $L / C$ : long and sometime concavo-convex; $Q$ : all quartz but not quartzite; F: feldspars and granite fragments; R: Other rock fragments; in this study, $R$ consists mainly of volcanic and metamorphic fragments); Visible porosity is not including micropores

\section{III.2. Diagenesis}

The clay minerals were contemporarily deposited with the detrital materials in syndepositional period so called matrix clays. Besides, the clay minerals that are created in diagenesis are a role as cement, sticking grains together. The analyzed clay minerals by XRD examination were extracted from Oligocene sandstones of the oil fields in study area (fig. 1).

The diagenesis of the sandstones is progressively increasing following the burial depth as showing the amount of kaolinite is decreasing whereas the volume of chlorite and illite are greater from sequence BH1.2 to sequence E1 (tab. 5). Smectite just presents in sequence BH1.2 at the west edge of basin but completely absents from sequence $\mathrm{C} 1$. Appearing mixed layers of illitesmectite tends increasing at deeper burial. This reveals the temperature is lightly increasing from the west part to the center of basin. At the higher burial with increasing the temperature kaolinite transfers into dickite, which is large euhedral crystals arranging face to face as booklets form, and into zeolite, which is in pore-filling form owing to the effect of hydrothermal flow extracted from underlain igneous basement.

Additionally, the sandstones are heavily mechanically squeezed and dissolved through sequences and the framework grains of the sandstones in sequence BH1.2 and sequence C1 contact each other by point to point and long types. The contacts change into long and concavo-convex and feldspar, volcanic fragments are strongly leached, forming secondary porosity in sequence E1. Diagenetic processes result to decrease the porosity and permeability of the sandstones, thus the porosity and permeability of the sandstones in sequence $\mathrm{E} 1$ are smaller than those of the sandstones in above sequences but the porosity is partly enhanced by the secondary porosity.

Table 5. Semi-quantitative percentage of clay fraction

\begin{tabular}{|c|c|c|c|c|c|c|}
\hline \multirow{2}{*}{ Sequence } & \multirow{2}{*}{ Range } & \multicolumn{5}{|c|}{ Semi-quantitative percentage of clay fraction (\%) } \\
\cline { 3 - 7 } & & Kaolinite & Chlorite & Illite & Smectite & $\begin{array}{c}\text { Mixed layers of } \\
\text { Illite-Smectite }\end{array}$ \\
\hline \multirow{2}{*}{ BH1.2 } & Min-Max & $38.7-88.0$ & $7.2-56.9$ & $2.2-26.3$ & $0.0-30.2$ & $0.0-22.0$ \\
\cline { 2 - 7 } & Common & $40.0-61.5$ & $20.0-57.4$ & $6.1-27.1$ & $16.2-22.6$ & $2.0-4.2$ \\
\cline { 2 - 7 } & &
\end{tabular}


Lieu, Kim Phuong.et.al. Int. Journal of Engineering Research and Application ISSN : 2248-9622, Vol. 7, Issue 6, ( Part -7) June 2017, pp.62-73

\begin{tabular}{|c|c|c|c|c|c|c|}
\hline \multirow{2}{*}{ C1 } & Min-Max & $23.9-75.0$ & $4.0-67.8$ & $4.3-29.5$ & 0.0 & $0.0-19.0$ \\
\cline { 2 - 7 } & Common & $34.0-58.8$ & $18.0-59.1$ & $6.2-24.8$ & 0.0 & $6.0-16.0$ \\
\hline \multirow{2}{*}{ D1 } & Min-Max & $32.7-68.3$ & $16.0-69.9$ & $12.2-45.5$ & 0.0 & $0.0-14.0$ \\
\cline { 2 - 7 } & Common & $33.2-52.5$ & $20.6-62.2$ & $13.0-27.8$ & 0.0 & $3.8-9.0$ \\
\hline \multirow{2}{*}{ E1 } & Min-Max & $22.7-38.2$ & $6.0-100.0$ & $14.5-79.5$ & 0.0 & $0.0-31.8$ \\
\cline { 2 - 7 } & Common & $24.2-32.6$ & $25.0-68.8$ & $18.0-57.7$ & 0.0 & $3.5-10.4$ \\
\hline
\end{tabular}

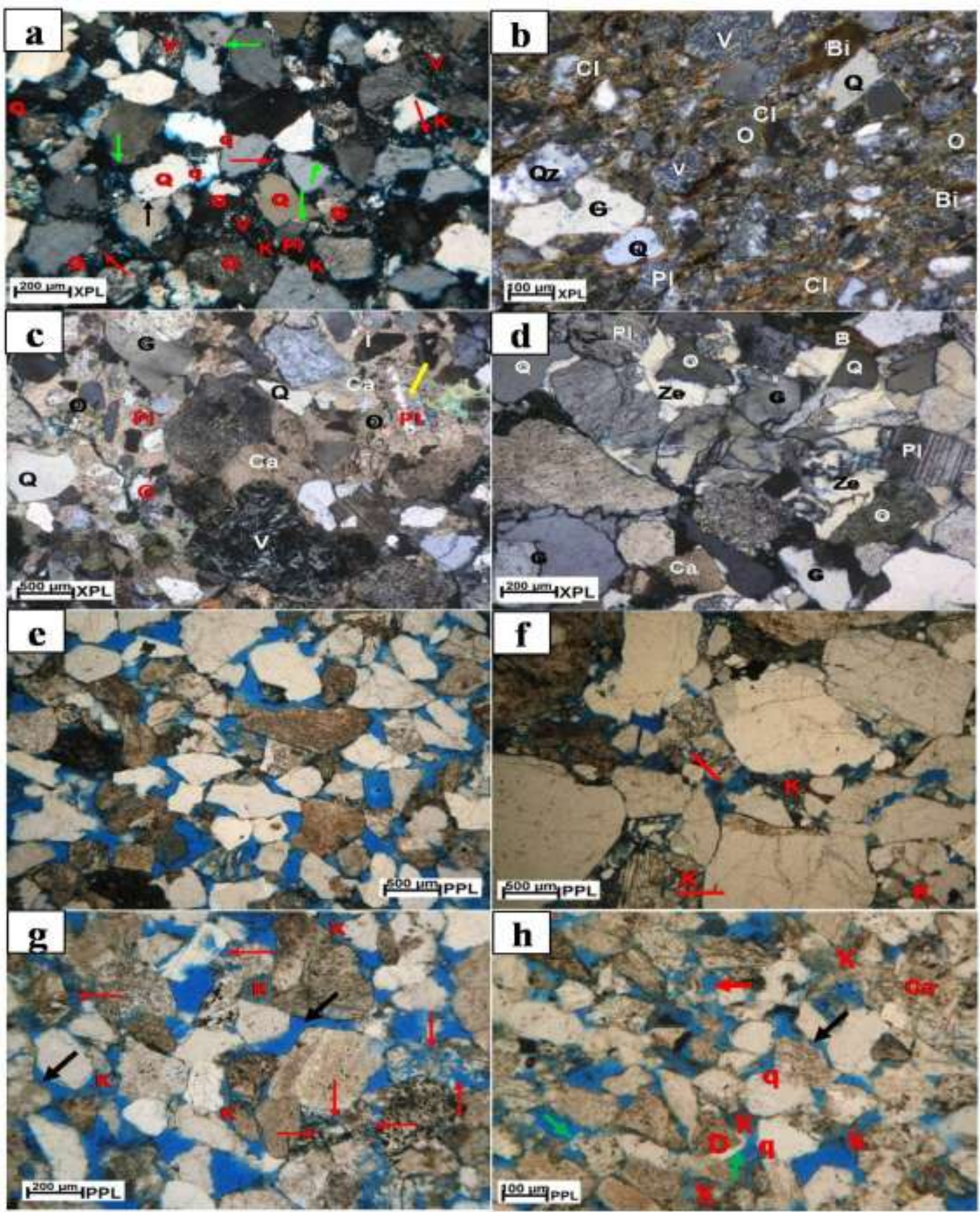

Figure 2. Photomicrographs of Oligocene sandstones. The major composition of sandstone is quartz $(\mathrm{Q})$, plagioclase $(\mathrm{Pl})$, orthoclase $(\mathrm{O})$, biotite $(\mathrm{Bi})$ and lithic fragments as granite $(\mathrm{G})$, quartzite $(\mathrm{Qz})$, volcanic debris $(\mathrm{V})$. 
(a) sandstone from $3057.84 \mathrm{~m}$ depth in sequence $\mathrm{C} 1$ is cemented by clays (green arrows), kaolinite $(\mathrm{K}$, red arrows) filling in pores in local quartz overgrowths (q) grow on quartz surface; (b) clay matrix-rich $(\mathrm{Cl})$ sandstone from $3314.5 \mathrm{~m}$ depth in sequence D1; (c\&d) sandstones from $3473.5 \mathrm{~m}$ and $3456.9 \mathrm{~m}$ in sequences $\mathrm{E} 1$ are cemented by calcite (Ca) in poikilotopic form and zeolite (Ze). Yellow arrow indicates calcite replaces in plagioclase; (e) moderately-well sorted sandstone from $2756.82 \mathrm{~m}$ depth in sequence BH1.2 is good porosity (stained blue color); (f) poorly sorted sandstone from $3053.22 \mathrm{~m}$ depth in sequence $\mathrm{C} 1$ is in poor porosity. Sometimes, kaolinite fills in pores and red arrows indicate feldspars dissolved forming secondary pores; (g) medium grained sandstone from $3041.85 \mathrm{~m}$ depth in sequence $\mathrm{C} 1$ is in good permeability with wide pore throats (black arrows); (h) fine grained sandstone from $3034.33 \mathrm{~m}$ depth in sequence $\mathrm{C} 1$ has moderate-poor permeability with narrow pore throats (black arrow) sporadically quartz overgrowths (q, green arrows) cover on quartz surfaces.

III.3. Features impact on the porosity and permeability of sandstone reservoir rocks

The porosity and permeability of sandstone are reduced during lithic formation owing to the influences of geological age, tectonic, depositional environment, etc. However, the study just considers the basic properties recognized by thin section analysis as mineralogical composition, grain texture, compaction, cementation in postdepositional processes.

The major composition of the sandstones is dominated by quartz, feldspar including $\mathrm{K}$ feldspar and plagioclase, lithic fragments like dominant granite, volcanic debris and a lesser quartzite, schist and chert. The composition lessly affects on the porosity and permeability of the sandstones in this study. The profound influence on the porosity of these sandstones is realized by grain texture such as grain size and grain sorting as shown in (fig. 2e\&f) and (fig. 3 -6). Figures show that the porosity of both the very fine-fine and medium-coarse grained sandstones is decreasing as the grain sorting is worse. The poorly sorted sandstones have high proportion of matrix to detrital grains and the finer grains of matrix fill in pore spaces between framework grains, thus the porosity is inhibited. The porosity of the mediumcoarse sandstones are larger than that of the very fine-fine ones. This may be because the finer sandstones have suffered more compaction. However, some of R-squared values on chart are low because the identical samples for comparison are few. The correlation between grain texture and porosity of the carbonate and zeolite cemented sandstones is not analyzed because their porosities are very poor or sometimes non existence.

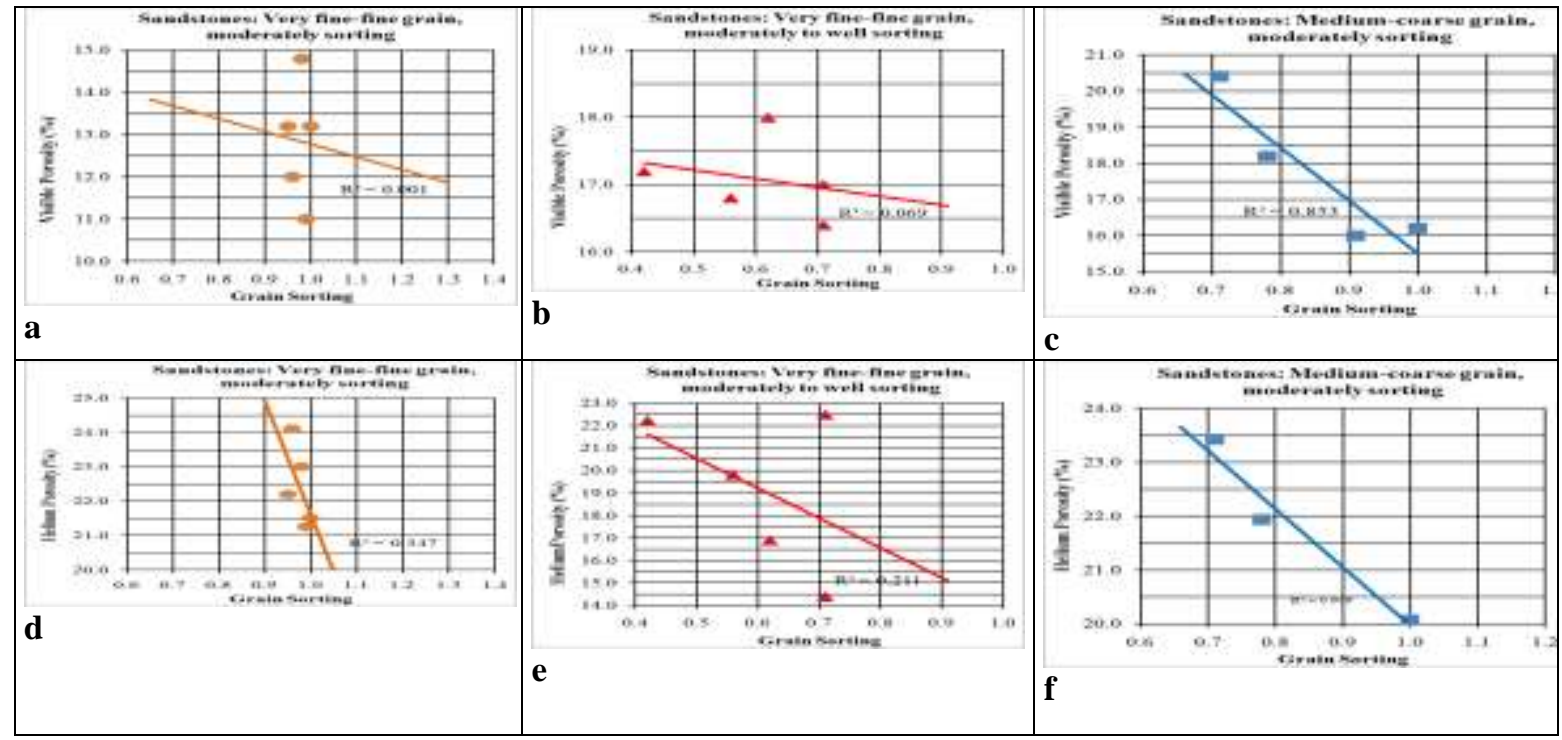

Figure 3. Correlation between grain size, grain sorting and porosity of sequence BH1.2

The visible porosity is not including micropores (fig. $3 \mathrm{a}-\mathrm{c}$ ) and helium porosity (fig. 3 d-f). Figure $3 \mathrm{~b}$ shows that the decline trend of porosity is not sharp compared to the other figures because the determined samples are not in a similar condition. 


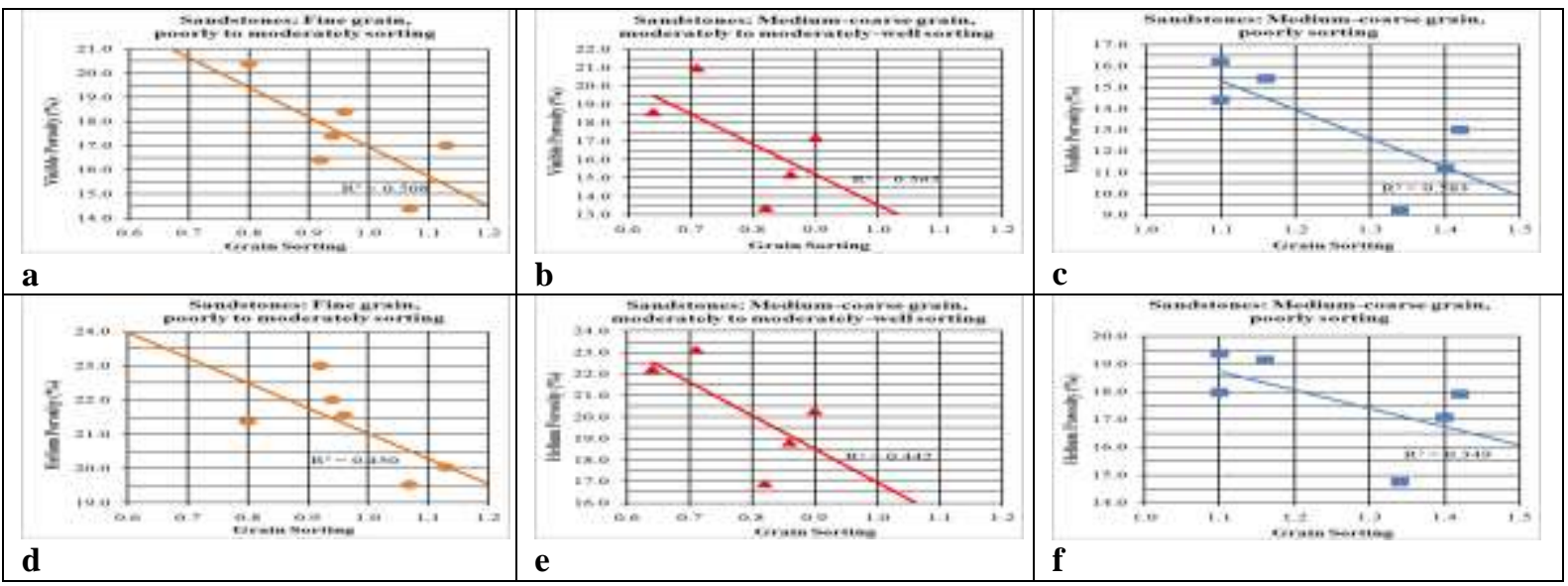

Figure 4. Correlation between grain size, grain sorting and porosity of sequence $\mathrm{C} 1$

The visible porosity is not including micropores (fig. 4 a-c) and helium porosity (fig. 4 d-f)

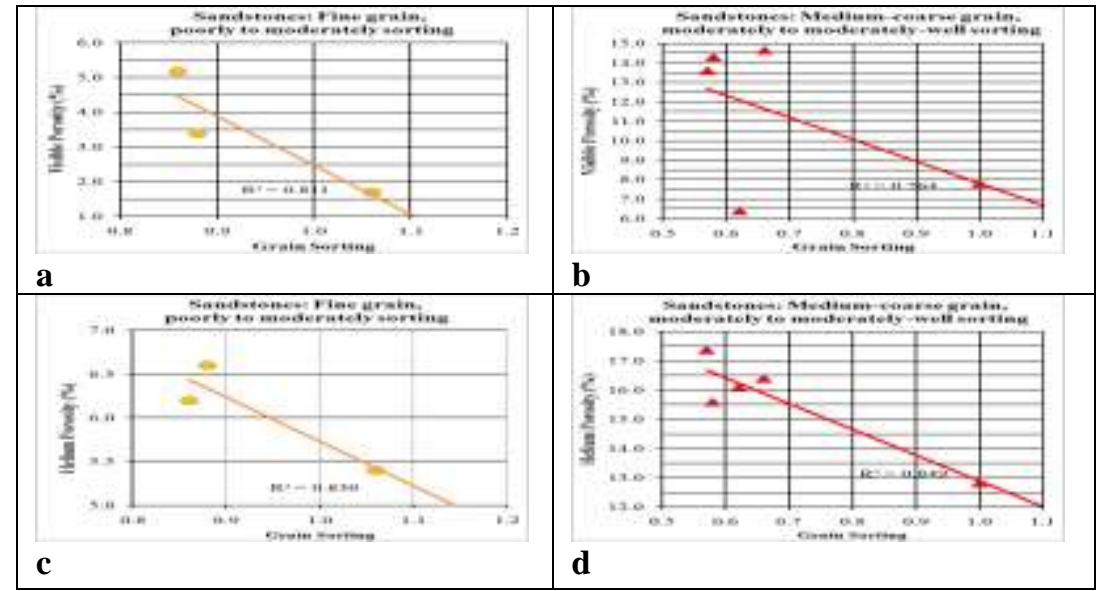

Figure 5. Correlation between grain size, grain sorting and porosity of sequence D1

The visible porosity is not including micropores (fig. $5 \mathrm{a \& b}$ ) and helium porosity (fig. $5 \mathrm{c} \& d$ )

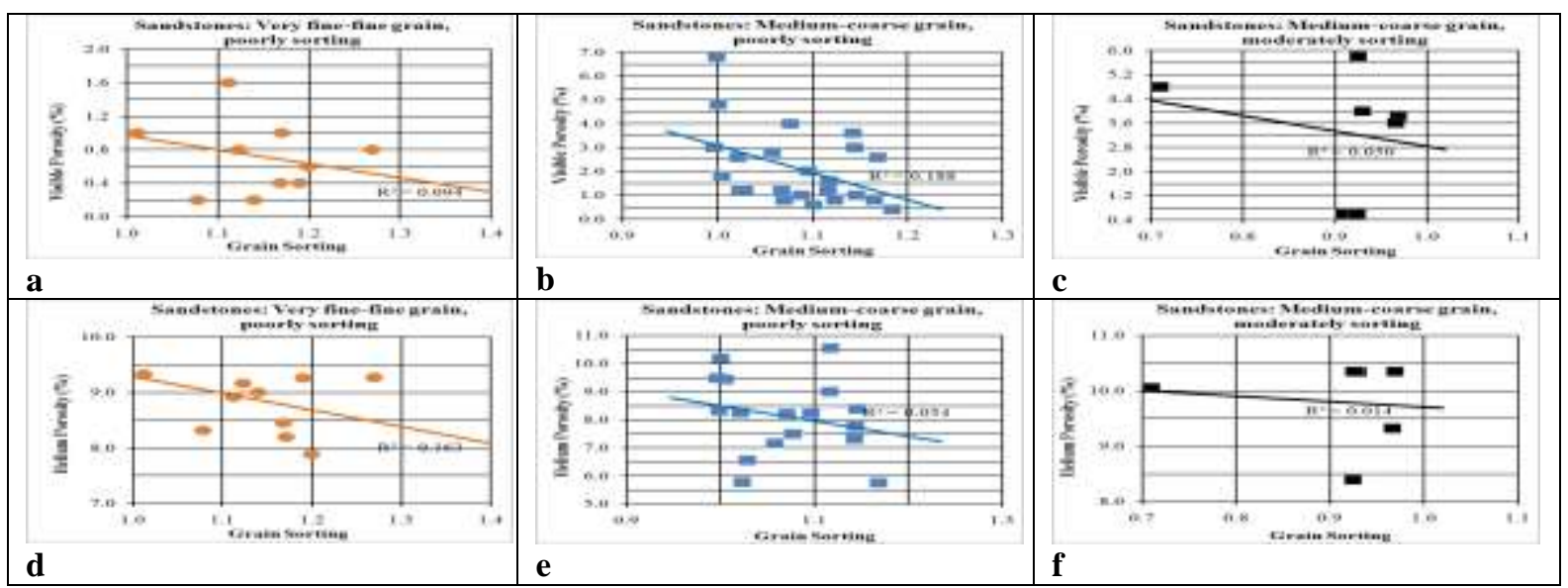

Figure 6. Correlation between grain size, grain sorting and porosity of sequence E1

The visible porosity is not including micropores (fig. $6 \mathrm{a}-\mathrm{c}$ ) and helium porosity (fig. $6 \mathrm{~d}-$ f). Figure $6 \mathrm{f}$ reveals that the decline trend of porosity is not sharp compared to the other figures because the determined samples are not in a similar condition.

Additionally, the permeability is particularly impacted by the grain size. The coarse grained 
sandstones have wide pore throats and fluid easily flows through pore spaces so their permeability is larger than that of the finer grained sandstones, which have narrow pore connections (fig. $2 \mathrm{~g} \& \mathrm{~h}$ ), (tab. 2, $3 \& 4)$. The sandstones containing abundant matrix have a lower porosity and permeability compared to those of the clean sandstones owing to pore-filling matrix (fig. 2b). Besides, the permeability is strongly correlated to the porosity that means the large porosity is corresponding to the large permeability. The good sandstone reservoir capacity must have the highly porosity and permeability, in order to hold the huge fluid volume in which fluid flows easily through pore spaces of rocks. The relation between the porosity and permeability of the analyzed sandstones reveals that the higher porosity is the higher permeability as shown in figure 7 .

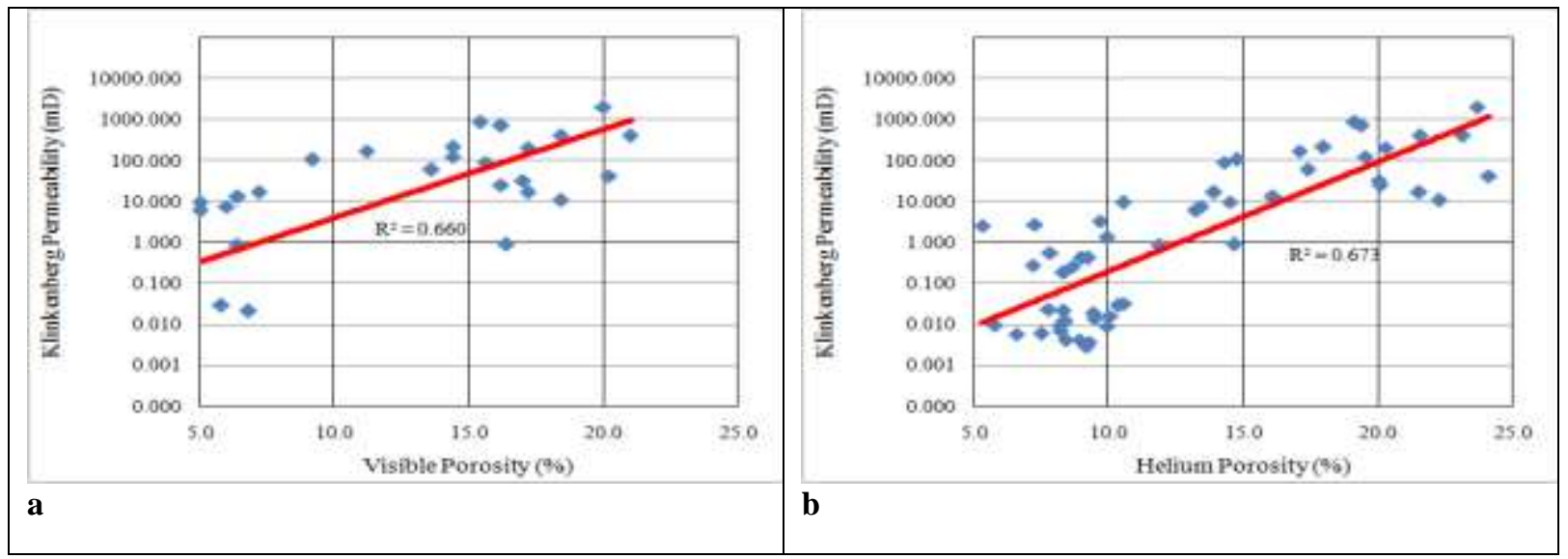

Figure 7. Correlation between porosity and permeability

With the deeper burial, cementation and mechanical compaction significantly control on the porosity of sandstone, as shown in figure 8 . Oligocene sandstone reservoir rocks are cemented by carbonate, zeolite and clays. The sandstones in sequences BH1.2 and C1 are weakly cemented and the destroyed intergranular porosity, which is visible porosity under microscope, is due to cementation $(10-40 \%)$. At the deeper depth the strong cementation takes place in sequences D1 and E1 and the intergranular porosity is lost from $20 \%$ up to $97 \%$. The sandstones in sequence E1 are seriously cemented by zeolite. The sandstones are squeezed increasingly along with the burial as showing the grain contacts changes from point to point and long types in sequences $\mathrm{BH} 1.2$ and $\mathrm{C} 1$ into long and concavo-convex types in sequences D1 and E1. The compaction is harmful for the porosity of the sandstones in sequences BH1.2 and C1 roughly 15$75 \%$ and $25-80 \%$ in sequences D1 and E1. It demonstrates that the porosity of the sandstones in sequences $\mathrm{BH} 1.2$ and $\mathrm{C} 1$ is better preserved. In particular, the secondary porosity formed owing to the dissolution of feldspar and volcanic debris is rising following the burial depth of the sandstones in sequences D1 and E1 (fig. 9a).

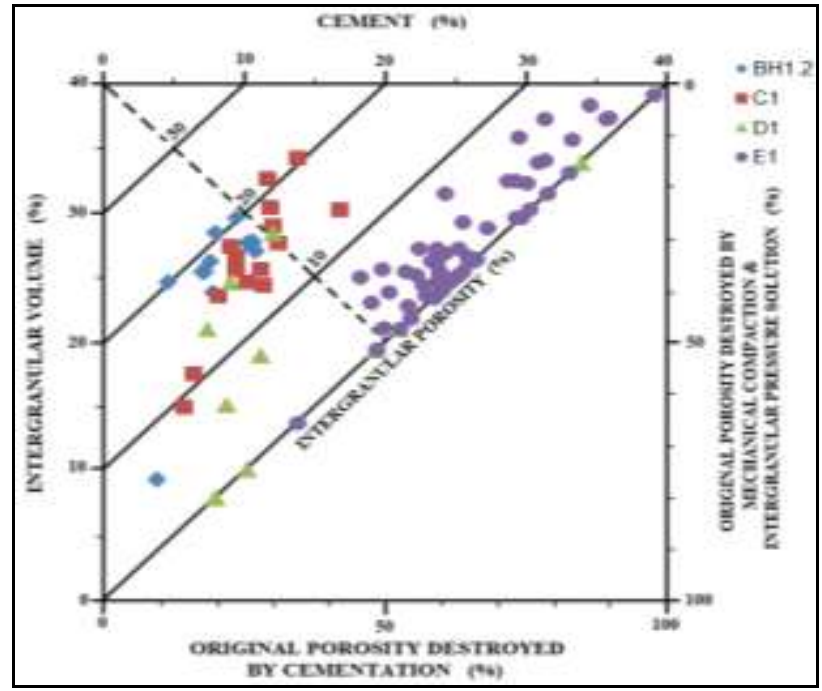

Figure 8. The intergranular porosity lost due to cementation and compaction (Houseknecht, 1987) 
Clay minerals that are either in pore-filling or in grain surrounding coatings have negative effects on the porosity and permeability. Most of the determined sandstones are clean, containing less than $15 \%$ of clay matrix and the total clay amount in percent composition of sandstone is partly declining the porosity (fig. 10a\&b).

Depending on their morphologies, the clays are significantly decreasing the permeability. Kaolinite is frequently in form of pore-filling aggregations with pseudohexagonal plates arranging as vermiforms and booklets that builds the complex pore structure. However, between kaolinite aggregations have micro pores that fluid can flow through pores (fig. 9b\&c). Hence kaolinite does not much reduce the permeability (fig. 11a). Illite and chlorite that appear as a mat coating on the quartz surface are responsible to prevent the growth of quartz overgrowths and tend to conserve the porosity of the sandstones (fig. 9d). Illite also occurs in pore-bridging ribbon and porefilling forms (fig. 9e\&f). In the work, illite is much abundant in pore-bridging form resulting to decline fluid flows through pores and has a great effect on the permeability (fig. 11b). Chlorite with irregular morphology arranges sub-parallel and grows perpendicular to detrital grain surfaces extending to pore spaces (fig. $9 \mathrm{~g} \& \mathrm{~h}$ ), in which chlorite is more abundant in perpendicular to detrital grain surface form and this is a reason to reduce significantly the permeability (fig. 11c). Smectite easily transfers into illite as the temperature increases and less frequently appears in oil fields so the study does not mention its effects.

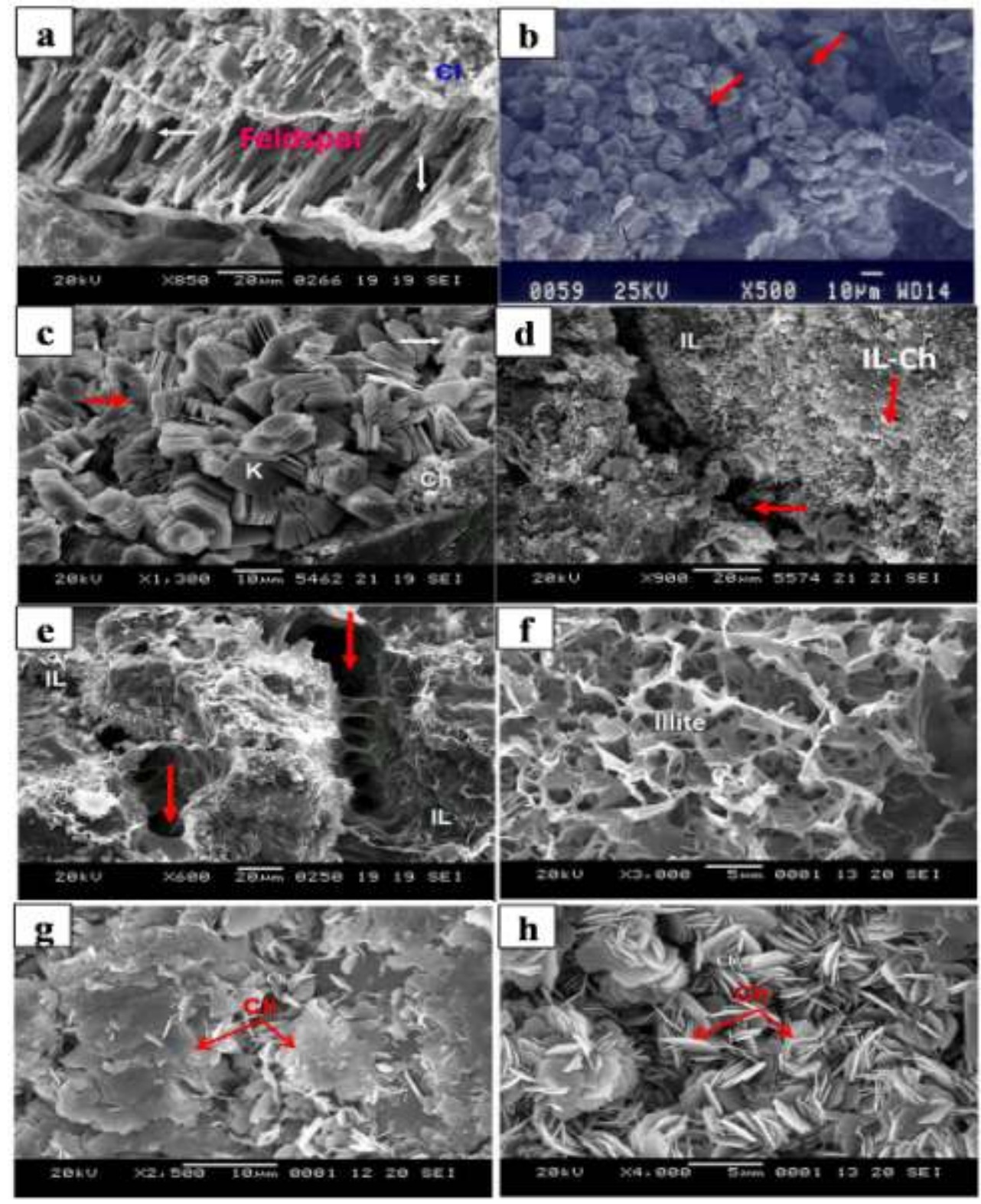

Figure 9. SEM images show the morphologies of clay minerals. 
(a) at $3404.04 \mathrm{~m}$ depth in sequence E1, feldspar is leached forming micro secondary pores (white arrows) and clay $(\mathrm{Cl})$ attaches on its edge; (b\&c) at $2177.1 \mathrm{~m}$ and $3398 \mathrm{~m}$ depths in sequences BH1.2 and E1, kaolinite and dickite in vermiforms and booklets fill in pores, red arrows indicate micro pores between kaolinite aggregations; (d) at $3057.84 \mathrm{~m}$ depth in sequence $\mathrm{C} 1$, illite (Il) and illite- chlorite (Il-Ch) cover on quartz surface and red arrow indicates pore space between grains; (e\&f) at $3313 \mathrm{~m}$ and $3406 \mathrm{~m}$ depths in sequence E1, illite is in porebridging form (red arrows) and detrital grain coatings; (g\&h) at 4290 and $4297.5 \mathrm{~m}$ depths in sequence E1, chlorite (Ch) arranges sub-parallel and grows perpendicular to detrital grain surfaces.

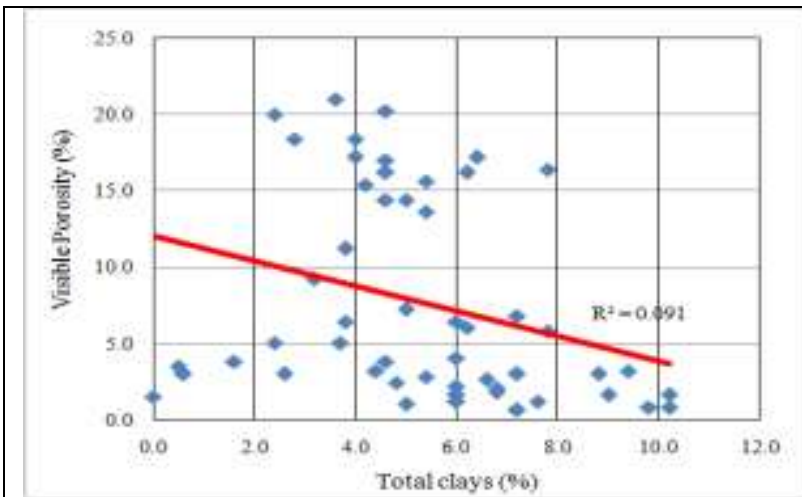

a

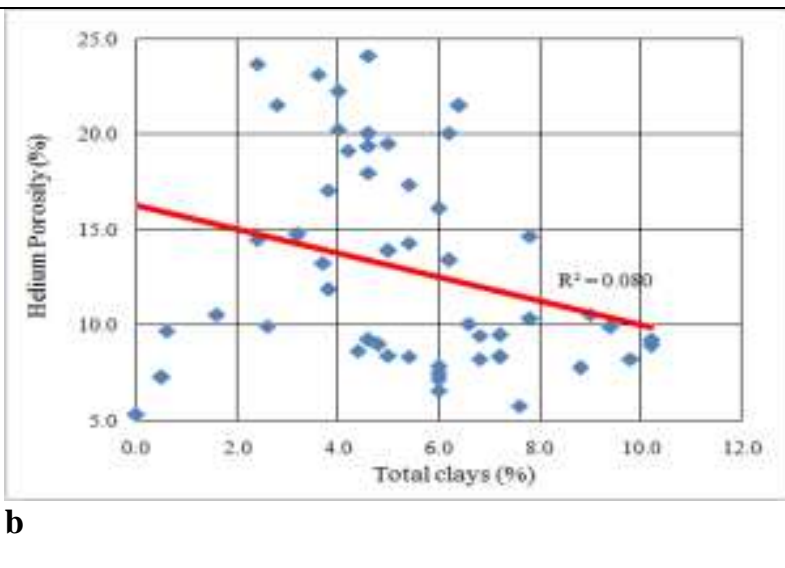

Figure 10. Correlation between total clays and porosity

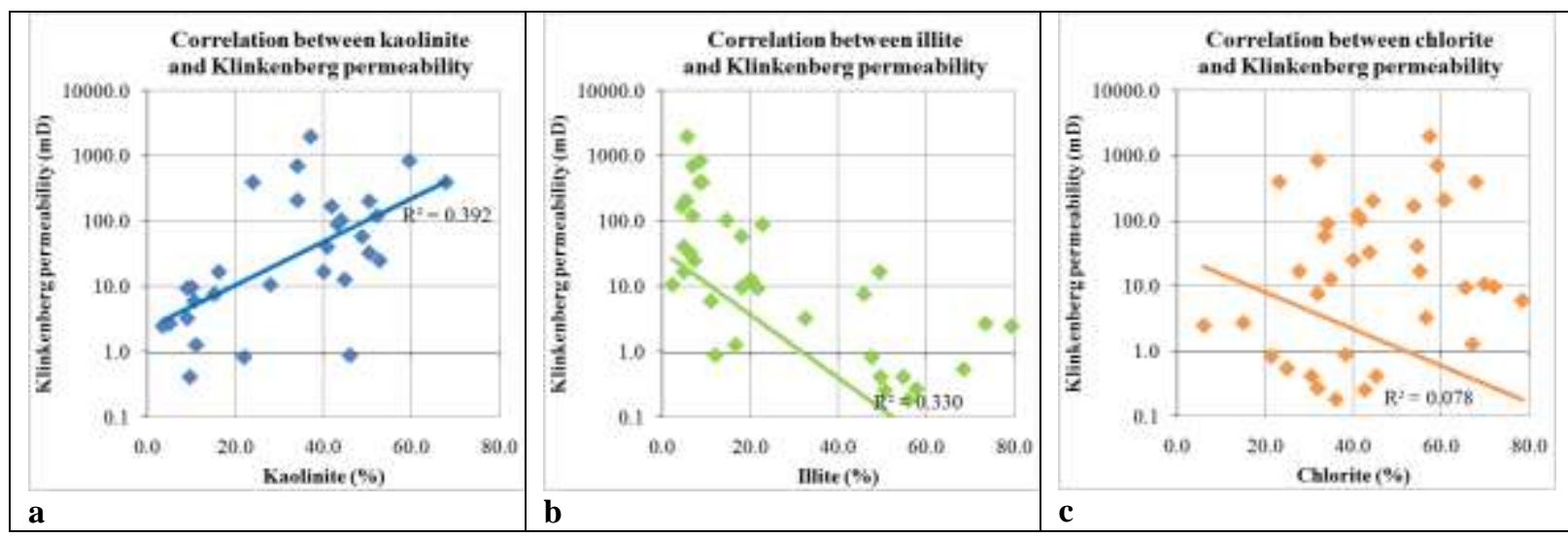

Figure 11. Correlation between clay fraction and permeability

\section{DISCUSSION AND CONCLUSIONS}

The analyzed Oligocene sandstone reservoir rocks of block 15-2 in Cuu Long basin are classified mainly arkose, lithic arkose that have the very poor to excellent porosity, infrequently interbedded by feldspathic greywacke and carbonate cemented sandstones with very poor porosity in sequences BH1.2 and C1. Particularly, the matrix-rich sandstones and the zeolite cemented sandstones dominantly present in sequences D1 and E1 respectively. The grain sorting and grain size have serious effects to the porosity and permeability of the sandstone. The moderately-well sorted, mediumcoarse grained sandstones have the best both porosity and permeability. This is consistent to figures illustrated by Selle (2000). The fine grained sandstones have undergone more compaction and at the high burial depth the sandstones are more heavily cemented and squeezed. Illite and chlorite have a profound effect on the permeability.

In brief, the sandstones in sequences BH1.2 and C1 are the best storage rocks in Oligocene sandstone strata.

\section{ACKNOWLEDGMENTS}

We gratefully acknowledge VPI-Labs for supporting the laboratory to carry out this investigation. We thank Mr. Nguyen Van Dung (ITC-PVEP) for his helpful suggestions and discussions in our research.

\section{REFERENCES}

[1]. Boyle, R. (1660). New Experiments PhysicoMechanicall, Touching the Spring of the Air, and its Effects (Made, for the Most Part, in a 
New Pneumatical Engine) Written by Way of Letter to the Right Honorable Charles Lord Vicount of Dungarvan, Eldest Son to the Earl of Corke. Oxford: H. Hall.

[2]. Bear, J. (1972). Dynamics of fluids in porous media. Dover Publications, New York Folk, R. L. (1974). Petrology of Sedimentary Rocks. Austin, Texas 78703, Hemphill Publishing Company.

[3]. Folk, R.L., Andrews, P.B. and Lewis, D.W. (1970). Detrital sedimentary rock classification and nomenclature for use in New Zealand. New Zealand Journal of Geology and Geophysics, v. 13, p. 937-968.

[4]. Griffiths, J. C. (1952). Grain size distribution and reservoir rock characteristics. American Association of Petroleum Geologist Bulletin, v. 36, no. 2, p. 205.

[5]. Henry D, Henry PG (2003). Darcy and other pioneers in hydraulics: Contributions in celebration of the 200th birthday of Henry Philibert Gaspard Darcy. Pa. ASCE Publications, Philadelphia Houseknecht, D.W. (1987). Assessing the relative important of compaction processes and cementation to reduction of porosity in sandstones. American Association of Petroleum Geologist Bulletin, v. 71, no. 6, p. 633-642.

[6]. Klinkenberg, L. J. (1941). The permeability of porous media to liquids and gases. Drilling and production practice. American Petroleum Inst, p. 200-213

[7]. Levorsen, A. I. (1967). Geology of Petroleum. $2^{\text {nd }}$ edition, San Francisco - London.

[8]. Mai Hoang Dam and Chu Duc Quang (2015). Sequence stratigraphy and determination of Early Miocene - Oligocene sedimentation environment of block 09-3 in Cuu Long basin based on the characteristic of freshwater dinocyst group and palynofacies analysis. Petro Vietnam, no. 7: p. 24-32.

[9]. McKinley, J. M., Atkinson, P. M., Lloyd, C. D., Ruffell, A. H., Worden, R. H. (2011). How Porosity and Permeability Vary Spatially With Grain Size, Sorting, Cement Volume, and Mineral Dissolution In Fluvial Triassic Sandstones: The Value of Geostatistics and Local Regression. Sedimentary Research, v. 81, no. 12, p. 844-858.

[10]. Morad, S. (1998). Carbonate Cementation in Sandstones: International Association of Sedimentologists, Special Publication 26, p. 126.

[11]. Pettijohn, F. J., Potter, P. E. and Siever, R. (1987). Sand and sandstone. Springer-Verlag Berlin. Heidelberg.

[12]. Selle, R. C. (2000). Applied Sedimentology. $2^{\text {nd }}$ edition, Academic Press, p. 74.
[13]. Tran Le Đong and Phung Dac Hai (2005). Cuu Long basin and Petroleum resource. Geology and Vietnam petroleum resource. Ha Noi, Vietnam petroleum Corporation, p. 271-314.

[14]. Welton, J. E. (2003). SEM Petrology Atlas. American Association of Petroleum Geologist Tulsa, Oklahoma 74101, USA, no. 4, p. 9-96.

[15]. Wentworth, C. K. (1922). A scale of grade and class terms for clastic sediments. Journal of Geology.

[16]. Worden, R.H. (1998). Dolomite cement distribution in a sandstone from core and wireline data: the Triassic fluvial Chaunoy Formation, Paris Basin, in Harvey, P.K., and Lovell, M.A., eds., Core-Log Integration: Geological Society of London Special Publication 136, p. 197-211.

[17]. http://www.bruker.com. X-ray Diffraction and Elemental Analysis X-ray Diffraction XRD Software TOPAS version 4.2. Copyright (C). 1999, 2009. 